\title{
La compétence discursive au croisement de la médiation intralinguistique et de la compétence grammaticale : exemple de transposition d'un dialogue en texte narratif
}

\author{
The discursive competence at the crossroads of the intra- \\ linguistic mediation and the grammatical competence: \\ example of a transposition from a dialogue into a narration
}

\author{
Monika Grabowska \\ Université de Wrocław \\ monika.grabowska@uwr.edu.pl
}

\begin{abstract}
The aim of the paper is to study the impact of the instruction, focused either on the grammatical component (the indirect speech) or the discursive component (the transformation of a dialogue into narration) of the communication competence on the quality of intralinguistic mediation carried out by learners at higher levels of competence (from B2). We argue by the divergence between the productions resulting from the first and the second instruction a need to ensure a didactic transition from the exercise of indirect speech (work on form) to its actual discursive use (social practice), according to the principles of the action-oriented approach.
\end{abstract}

Keywords: discursive competence, grammatical competence, intralinguistic mediation, dialogue, narration

\section{OBJECTIFS DE LA RECHERCHE ET CADRE DE RÉFÉRENCE}

L'objectif du présent article est d'examiner la qualité de la compétence discursive aux niveaux supérieurs de l'apprentissage du FLE (à partir de B2) dans le cadre de l'activité linguistique de médiation, corrélée à une composante grammaticale très valorisée dans les cursus et examens scolaires, à savoir la maîtrise du discours indirect. 
La sortie récente du manuel Les discours directs et indirects dans la série "Grevisse langue française » (Callet, 2012) témoigne d'un intérêt pédagogique constant pour ce problème de grammaire. En cours de français langue étrangère (désormais FLE), il est présenté surtout sous son aspect technique, englobant les ajustements des embrayeurs, la subordination des complétives et des interrogatives indirectes, la concordance des temps et le répertoire des verbes introducteurs. De cette manière, étudiée au niveau des transformations phrastiques, la maîtrise du discours indirect alimente, certes, la compétence discursive qui, selon le Cadre européen commun de référence (2001), se rapporte tout d'abord à « la connaissance de l'organisation des phrases et de leurs composantes » (p. 96), mais néglige ses autres éléments, à savoir "la capacité à les maîtriser en termes: de thème/rhème, d'information donnée/information nouvelle, d'enchainement «naturel » $(. .$.$) , de$ cause/conséquence; la capacité de gérer et de structurer le discours en termes: d'organisation thématique, de cohérence et de cohésion, d'organisation logique, de style et de registre, d'efficacité rhétorique, de principe coopératif $(. .$.$) ; la capacité$ à structurer ; le plan du texte » (p. 96).

Par rapport à la médiation linguistique, le Cadre précise notamment ce qui suit (point 2.1.3, p. 18):

Participant à la fois de la réception et de la production, les activités écrites et/ou orales de médiation, permettent, par la traduction ou l'interprétariat, le résumé ou le compte rendu, de produire à l'intention d'un tiers une (re)formulation accessible d'un texte premier auquel ce tiers n'a pas d'abord accès direct. Les activités langagières de médiation, (re)traitant un texte déjà là, tiennent une place considérable dans le fonctionnement langagier ordinaire de nos sociétés.

Ainsi, nous avons voulu examiner, dans le cadre d'une activité de médiation intralinguistique, la capacité de transformation d'un discours direct en discours indirect. Nous mettrons notamment en évidence l'impact de la consigne sur la qualité de la médiation effectuée entre le texte dialogal et narratif, et nous l'analyserons sous l'angle de la compétence discursive de l'apprenant telle qu'elle a été définie cidessus d'après le Cadre, l'hypothèse sous-jacente étant que la médiation d'un texte authentique conservant des traces du discours oral, effectuée sur la base de transformations syntaxiques relativement bien assimilées (domaine de la compétence langagière), ne permet pas nécessairement à l'apprenant de produire un nouveau texte acceptable du point de vue discursif.

\section{MODALITÉS DE L’EXPÉRIENCE}

Dans une expérience conçue pour les besoins de la présente recherche, nous avons demandé aux étudiants de transformer par écrit le texte d'un dialogue authentique et contextualisé en texte narratif, une partie d'entre eux ayant reçu une con- 
signe focalisée sur la composante linguistique, l'autre partie, sur la composante discursive de la compétence de communication. L'activité a été soumise en juin 2016 aux étudiants de $\mathrm{III}^{\mathrm{e}}$ année de licence (désormais IIIL, niveau acquis selon le programme : B2) et de I ${ }^{\text {ère }}$ année de maîtrise (désormais IM, niveau supposé : C1) de philologie française de l'Université de Wrocław. Les deux groupes ont regardé un extrait du film Entre les murs de Laurent Cantet (2008) accompagné de la transcription (tirée de Mennesson, p. 14) de l'interaction verbale. Il s'agit d'un échange entre un professeur (François) et des collégiens :

François : Je vais faire une phrase avec « succulent ». [Il écrit au tableau] « Bill déguste un succulent cheeseburger ».

Cherif: C'est pas bon un cheeseburger, ça pue ! Pourquoi vous mettez « cheeseburger»?

François : Cela dit, justement! Puisque tu trouves que ça pue, tu penses donc que les cheeseburgers ne sont pas succulents.

Cherif: Je m'en fiche, mais en tout cas un cheeseburger, c'est pas bon.

François : Ouais, d'accord. Mais ce que je veux dire, c'est que pour deviner « succulent », ce que je viens de vous dire devrait vous mettre la puce à l'oreille.

Angélica : Monsieur, ça veut dire quoi ?

François : Ça veut dire quoi «quoi »?

Angélica : La puce machin truc.

François : «La puce à l'oreille »? « Ça devrait vous mettre la puce à l'oreille». Personne ne connaît cette expression ? Voilà, "mettre la puce à l'oreille », ça veut dire «donner un indice». Donc quand je dis que dans la mesure où Cherif trouve que les cheeseburgers puent, il pense qu'ils ne sont pas spécialement succulents. Ça devrait vous expliquer définitivement ce que veut dire « succulent».

Khoumba : Et pourquoi vous arrêtez pas de mettre des «Bill»?

François : «Débile»?

Khoumba : Pas « débile », mais «Bill», là ! [elle montre le tableau]. Toujours des noms bizarres.

François : C'est pas du tout un nom bizarre! C'est le nom d'un président américain récent, je te rappelle.

Khoumba : Pourquoi vous mettez pas « Aïssata » ou « Rachid » ou « Mohammed » ou ...

Esméralda : Vous mettez tout le temps des noms de patos.

François : C'est quoi des noms de ....?

Esméralda : De babtous.

François : Babtous quoi ?

Esméralda : C'est-à-dire de babtous, de Français, de «çaifran ».

François : Mais t'es pas Française toi, Esméralda?

Esméralda : Non, moi j’suis pas Française.

François : Ah bon ! J'étais pas au courant [ironique].

Esméralda : En fait, j'suis Française mais j'suis pas fière de l'être.

François : Moi, non plus je suis pas fier d'être Français.

Khoumba : Mais pourquoi vous mettez toujours des noms comme ça alors? 
François : Mais enfin Khoumba, tu comprends bien que si je choisis à chaque fois des prénoms en fonction des origines diverses qu'il y a dans cette classe, j'vais pas m'en sortir. Esméralda et Khoumba (en même temps) : Ben, changez un peu !

François : Alors, qu'est-ce que tu me proposes ? [à Khoumba]

Khoumba : Aïssata ! Euh ... Fatou, non ... euh ...

Esméralda et Khoumba (ensemble) : Aïssata!

Les étudiants ont ensuite été invités à procéder selon la consigne imprimée sur leur fiche, sans savoir qu'elle avait été conçue en deux variantes en nombre égal mais distribuées aléatoirement :

Consigne $n^{\circ} 1$ : L'action du film Entre les murs (2008) se passe dans un lycée multiethnique dans la banlieue de Paris. François, qui enseigne avec passion et enthousiasme le français, a des difficultés avec certains élèves. Regardez la scène, lisez la transcription du dialogue et transformez-le en discours indirect.

Consigne $\mathrm{n}^{0} 2$ : (...) Regardez la scène, lisez la transcription du dialogue et transformez-le en un récit.

Le texte de base a été choisi pour différentes raisons. Premièrement, il est authentique compte tenu des modalités de tournage du film dont il provient. En effet, les jeunes acteurs n'étaient pas censés reproduire les dialogues d'un scénario, mais improviser autour d'une situation-problème (Bégaudeau, Cantet, Campillo, 2008, p. 14). Au niveau linguistique, le texte n'est pas difficile: il correspond au niveau B2 de compétence (dans la version transcrite, peut-être même au niveau B1), et ne devait donc pas poser de problèmes de compréhension susceptibles d'éclipser les problèmes d'ordre pragmatico-discursif qui étaient l'enjeu de l'expérience. La seule expression supposée difficile est "mettre la puce à l'oreille », mais elle est justement expliquée dans la scène en question. Sinon, il y a aussi deux mots argotiques susceptibles de ne pas être connus des apprenants («babtous » et « patos»), mais nous les avons expliqués en bas de page. Certes, le langage des collégiens est familier et argotique, mais les étudiants sont confrontés à ces deux variantes du français lors des cours de FLE. D'autre part, eux-mêmes les utilisent dans leur communication entre pairs en polonais. Par conséquent, en leur demandant de transposer un texte non-standard par écrit, il est possible de tester leurs stratégies transversales de médiation dans un nouveau contexte. Deuxièmement, le dialogue est authentique dans la mesure où il n'a pas été « épuré » de ses marques de discours oral (hésitations, faux départs, anacoluthes, transformations phonétiques transcrites...). Il présente donc un intérêt didactique majeur puisque dans la pratique pédagogique, les apprenants ne sont pas forcément confrontés à la médiation écrite de textes «bruts », non aseptisés pour des besoins pédagogiques. Finalement, et c'est l'argument le plus important, l'activité reproduit le besoin authentique de raconter : un film, une scène, une conversation. 


\section{TRANSFORMATION DU DISCOURS DIRECT EN DISCOURS INDIRECT}

Le corpus se compose de 12 copies, dont 7 de IIIL et 5 de IM. Nous les avons étudiées :

- au niveau des structures phrastiques englobant, selon la terminologie de Biardzka (2009, p. 105), une séquence textuelle binaire composée de deux segments : didascalie + citation, et correspondant, dans la terminologie de l'analyse conversationnelle, à une intervention - la plus grande unité monologale (Traverso, 1996, pp. 33-34); et :

- au niveau des structures formées par les échanges, ces derniers étant définis comme «la plus petite unité dialogale (...) composée d'un nombre variable d'interventions émises par les différents interlocuteurs » (Traverso, 1996, p. 31).

Nous ne nous sommes pas attardée sur les fautes de grammaire, d'orthographe et de ponctuation (à moins qu'elles aient un impact important sur le plan pragmaticodiscursif).

Première constatation, les réponses des étudiants ont été rédigées plutôt au passé qu'au présent - ce qui n'était pas imposé dans la consigne. Deux interprétations de ce fait sont possibles: premièrement, la transposition d'une scène s'effectue de façon naturelle au passé ; deuxièmement, ce choix serait influencé par un facteur psycho-pédagogique (les étudiants répondant à une attente présupposée de l'évaluateur).

Deuxième constatation, les textes produits sont morcelés : ils contiennent parfois autant de paragraphes qu'il y avait d'interventions dans l'interaction. Nous avons notamment observé un grand souci d'exhaustivité dans la transposition, qui résulte probablement d'une exposition intensive à des exercices de transformation exigeant de tenir compte de chaque élément phrastique. Parallèlement nous avons noté relativement peu d'effort pour obtenir des macrostructures pragmatiques rendant compte des valeurs illocutoires ou perlocutoires des actes de parole contenus dans l'interaction, mais en faisant en même temps abstraction des éléments discursifs propres au registre familier oral et n'ayant aucun impact sur le contenu informationnel de l'énoncé.

Dans la suite de ce point, en suivant le fil du dialogue, nous étudierons quelques exemples symptomatiques des stratégies utilisées par les étudiants. Ils rendent compte de différentes difficultés discursives de la médiation effectuée.

I. François : Je vais faire une phrase avec «succulent». [Il écrit au tableau] «Bill déguste un succulent cheeseburger ».

(1) François a dit qu'il allait faire une phrase avec «succulent» et il a écrit que Bill dégustait un succulent cheeseburger. 
(2) François a dit qu'il allait faire une phrase avec « succulent ». «Bill déguste un succulent cheeseburger ».

Dans le cas de cette première intervention, nous avons observé deux stratégies de transformation. La première respecte les règles syntaxiques du discours indirect, mais mélange deux ordres énonciatifs différents, en situant «Bill» (qui appartient au métadiscours) sur le même plan que « François » (discours). La deuxième ne tombe pas dans ce piège et les distingue bien, mais l'auteur ne trouve pas de solution pour assurer la cohésion entre les deux phrases transformées, et juxtapose le métadiscours au discours.

\section{Cherif : C'est pas bon un cheeseburger, ça pue ! Pourquoi vous mettez « cheese- burger $» ?$}

(3) Cherif lui crie que c'est pas bon un cheeseburger et que ça pue.

Dans cette intervention, la majorité des reformulations se sont basées sur le type de phrase (exclamative), ce qui se traduit par le choix du verbe "crier». Aucune réponse n'a tenu compte de l'objection formulée dans la deuxième phrase, qui détermine la valeur illocutoire de l'intervention. Au niveau de la cohésion, nous avons remarqué que plusieurs transformations gardaient la cataphore du « cheeseburger », caractéristique de la langue orale, et qui (sans doute à l'insu de l'auteur) apparente la transformation effectuée au discours direct libre.

Une autre question que cet exemple pose pour l'évaluateur est aussi celle de l'adverbe de négation «ne», absent dans le registre familier et courant: faut-il pénaliser ou ne pas pénaliser son absence dans la transformation effectuée par écrit ? Ce type de dilemme n'apparait pas dans le cas des documents fabriqués et écrits, préférés dans la pratique de l'enseignement. Toutefois, dans la démarche actionnelle, les comptes rendus, proposés aux apprenants comme tâches d'apprentissage, pourraient, voire devraient, se faire aussi à partir de dialogues ou monologues oraux, qui sont souvent loin de respecter les règles du système écrit codifiés dans les grammaires normatives ou dans les grammaires de FLE. L'exemple de la première partie de la négation est révélateur du besoin de conceptualisation de différents niveaux de langue lors des activités à dominante linguistique pratiquées à partir de données authentiques et concerne évidemment aussi le discours indirect.

III. François : Cela dit, justement ! Puisque tu trouves que ça pue, tu penses donc que les cheeseburgers ne sont pas succulents.

(4) François a dit que cela disait, justement et que, puisque Cherif trouvait que ça puait, s'il pense donc que les cheeseburgers ne sont pas succulents.

Les transformations de l'intervention (III) prouvent généralement un manque de compétence pragmatique (difficultés manifestes à prendre en charge le début de 
l'intervention, impossible à rapporter autrement qu'à partir de la valeur illocutoire de l'énoncé), mais aussi un certain désarroi sur le plan syntaxique causé par la subordination à deux étages.

IV. François : Ouais, d'accord. Mais ce que je veux dire, c'est que pour deviner « succulent », ce que je viens de vous dire devrait vous mettre la puce à l'oreille.

(5) François s'est mis d'accord avec lui mais il a ajouté que ce qu'il voulait dire c'était que pour deviner « succulent» ce qu'il venait de leur dire devrait leur mettre la puce à l'oreille.

(6) François a exprimé que ce qu'il venait de dire devrait les mettre la puce à l'oreille.

(7) François a expliqué que ce qu'il venait d'écrire devrait leur mettre la puce à l'oreille pour deviner « succulent ».

La plupart des transformations de cette intervention s'apparentent à des nœuds gordiens : les propositions subordonnées s'y chevauchent au gré de la fantaisie de l'apprenant, témoignant ainsi des difficultés à reconstituer le schéma structural de la longue phrase de François, les interférences entre «ce que», «c'est que » et un deuxième «ce que » ne facilitant pas la tâche ; à cela s'ajoute la paronymie de «je veux dire » et «je viens de vous dire ». L'exemple (6) témoigne d'une bonne intuition de l'apprenant (mais le verbe «exprimer» est malencontreux, et le pronom «les » mal ajusté au changement d'instance énonciative). Par contraste, l'exemple (7) apparait comme remarquable.

\section{Angélica : Monsieur, ça veut dire quoi ?}

(8) Angélica a demandé quoi ça voulait dire.

(9) Angélica a posé la question a monsieur ce quoi ça voulait dire.

(10) Angélica a demandé ce que ça voulait dire en ajoutant quoi à la fin (...).

La transformation au discours indirect d'une des questions les plus simples, que les apprenants s'approprient dès le début du processus d'apprentissage d'une langue étrangère, et qui consiste à interroger sur le sens d'un mot nouveau, est extrêmement problématique, surtout si elle est exprimée dans le registre familier (l'exemple 10 étant le plus frappant de la série).

\section{François : Ça veut dire quoi « quoi »?}

(11) François lui a demandé quoi voulait dire « quoi ».

(12) (...) et François a demandé ce que ça voulait dire « quoi ».

(13) François lui a demandé de préciser car il n'a pas compris le « quoi » dans la question posée par Angélica.

(14) François a demandé de préciser la question.

Après toute une série de maladresses syntaxiques causées par le double statut du mot « quoi », comme dans l'exemple (11), les trois exemples suivants présentent des 
transformations correctes mais susceptibles d'être jugées différemment selon le critère d'acceptabilité discursive en fonction du registre : (12) est une simple transformation phrastique gardant le registre familier ; (13) est le plus explicite et ressemble à un discours diagnostique ; c'est seulement la phrase (14) qui résume de façon concise l'intention illocutoire de l'acte de parole de François.

\section{Angélica : La puce machin truc.}

(15) Angélica a repondu qu'il s'agissait de puce machin truc.

(16) Angelica lui a repondu que la puce était la chose.

(17) Angélica a dit qu'il s'agissait de l'expression avec le mot « puce ».

(18) L'élève lui a expliqué qu'elle ne comprenait pas la structure avec le mot « la puce ».

La transposition de la réponse pose un problème de taille à la plupart des étudiants, leur manque de compétence pragmatique entraînant une méprise sur le sens de cet énoncé (ex. 15). Seulement deux réponses assurent la progression thématique du texte : (17) et (18); (16) témoigne d'une bonne intuition, mais l'expression est maladroite car l'expression de départ était verbale («mettre la puce à l'oreille »).

VIII. François : «La puce à l'oreille »? «Ça devrait vous mettre la puce à l'oreille ». Personne ne connaît cette expression ? Voilà, « mettre la puce à l'oreille », ça veut dire « donner un indice». Donc quand je dis que dans la mesure où Cherif trouve que les cheeseburgers puent, il pense qu'ils ne sont pas spécialement succulents. Ça devrait vous expliquer définitivement ce que veut dire « succulent ».

(19) François s'étonnait que personne ne connaissait l'expression «ça devait vous mettre la puce à l'oreille ». Il a expliqué que ça voulait dire « donner un indice ». Il a ajouté que quand il disait que dans la mesure où Cherif trouvait que les cheeseburgers puent, il pensait qu'ils ne sont pas spécialement succulents. Il a dit que ça devrait leur expliquer définitivement ce que voulait dire « succulent».

(20) François s'est rassuré s'il s'agissait de la puce à l'oreille et il a précisé l'expression que ça devrait leur mettre la puce à l'oreille. (...)

(21) François s'assure qu'elle pense de la puce à l'oreille. Il dit sur exemple que cela devrait les mettre la puce à l'oreille. (...)

(22) François a répété l'expression et a demandé aux élèves si personne ne connaissait cette expression. (...)

(23) François a demandé si c'était l'expression « la puce à l'oreille » et il a exclamé une autre expression avec ce mot : « Ça devait mettre la puce à l'oreille ». (...)

(24) François s'assure si elle pense à l'expression « la puce à l'oreille ». Il dit une phrase exemplaire : «Ça devrait vous mettre la puce à l'oreille». Il demande si personne ne connaît cette expression-là et il explique que «mettre la puce à l'oreille » veut dire «donner un indice» et que quand il dit que dans la mesure où Cherif trouve que les cheeseburgers puent, ce garçon pense qu'ils ne sont pas spécialement succulents. François ajoute que cela devrait leur expliquer définitivement ce que veut dire « succulent ». 
La plupart des réponses sont défectueuses du point de vue syntaxique. La phrase de départ : «Donc quand je dis que dans la mesure où Cherif trouve que les cheeseburgers puent, il pense qu'ils ne sont pas spécialement succulents » constitue pour plusieurs étudiants une difficulté insurmontable du fait de l'auto-citation de François et d'une erreur de transcription dans le document qui leur a été distribué, ce dont nous ne nous sommes rendu compte qu' a posteriori (la proposition principale « Ça devrait vous expliquer définitivement ce que veut dire "succulent" » y est présentée comme une phrase autonome). Les exemples (19-22), en dépit de leur syntaxe défectueuse, témoignent d'une volonté de résumer la valeur illocutoire de l'énoncé grâce au choix des verbes introducteurs: «se rassurer», «s'assurer», «s'étonner», « répéter l'expression »; * «exclamer » (probablement pour «s'exclamer ») de (23) serait en revanche un mauvais exemple. L'exemple (24) est le mieux élaboré du point de vue discursif, sans pour autant éviter le piège du besoin de restituer la proposition principale par rapport à « quand il dit que... ».

\section{Khoumba : Et pourquoi vous arrêtez pas de mettre des « Bill »?}

\section{François : «Débile »?}

(25) Khoumba a demandé pourquoi il arrêtait pas de mettre des «Bill» et François a demandé s'il disait « débile ».

(26) (...) François lui a demandé s'il disait « débile ».

(27) Khoumba a demandé pourquoi il arrêtait pas de mettre des «Bill ». François s'est rassuré s'il s'agissait de débile.

(28) Khoumba demande pourquoi il n'arrête pas à mettre des «Bills ». François demande si elle a dit « débile».

(29) Khoumba a posé une question pourquoi le professeur arrêtait pas de mettre des « Bill ». François a dit « Débile».

(30) (...). François a demandé s'il s'agissait d'un mot « débile».

(31) Khoumba, une autre élève a posé la question, pourquoi il n'arrêtait pas de mettre des «Bill ». François a mal entendu, il répète « Débile ? ».

(32) Khoumba a demandé pourquoi François n'arrêtait pas de mettre des «Bill» dans les exemples de phrases sur le tableau. François s'est étonné d'entendre « débile».

Certains étudiants n'ont pas observé de quel sexe était l'élève Khoumba, ce qui dans leur production aboutit à une erreur pragmatique, comme dans (25) et (26). D'autres réponses ne rendent pas compte de façon pertinente de l'acte de parole : le verbe «se rassurer » de (27) est plus précis (mais inadapté au contexte) que « dire », acceptable dans (28) plus que dans (29), mais pas dans (26) à cause de l'imparfait à la place du plus-que-parfait. En plus, dans (27), l'étudiant mélange les plans énonciatifs en enlevant les guillemets autour du mot « débile». Dans (30), l'article indéfini gâche l'effet obtenu en introduisant une hésitation pragmatique puisque le mot est bel et bien cité. L'exemple (31) est remarquable par le souci d'introduire une nouvelle chaîne de références à travers le complément du nom « une autre élève », 
ainsi qu'une didascalie supplémentaire (même si elle n'est pas forcément juste) : «François a mal entendu ». Finalement, l'exemple (32) est proche de l'idéal ${ }^{1}$.

\section{Khoumba : Pas « débile », mais « Bill », là ! [elle montre le tableau]. Toujours des noms bizarres.}

(33) [Khoumba a demandé pourquoi François n'arrêtait pas de mettre des «Bills ».] Il a demandé pourquoi François mettait toujours des noms bizarres.

(34) Cette question dérange une élève, qui exclame : «Pas "débile", mais "Bill", là !». Elle montre le tableau. Elle se plaigne que toujours existaient des noms bizarres.

(35) Khoumba a expliqué qu'il s'agissait du prénom «Bill» se trouvant au tableau. Elle a montré le tableau en se plaignant que le prof mette toujours des noms bizarres.

Dans cette série, nous pouvons observer une énorme réticence à insérer des éléments provenant de l'énonciateur et susceptibles d'assurer une meilleure cohésion du dialogue transposé. Les transformations, proches du texte de départ, s'effectuent à travers la conjonction de coordination « et » ou par des juxtapositions de structures phrastiques parallèles (exemple 33 notamment). Le souci de mettre en valeur l'enjeu de l'interaction, même s'il est maladroit dans l'expression, est visible dans (34), à travers les performatifs «cette question dérange un élève », * «elle exclame »,

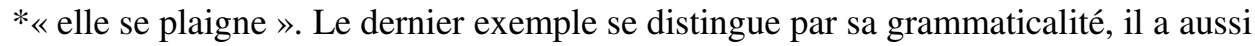
le mérite de classer le mot «Bill» parmi les prénoms en contribuant ainsi à une reformulation cohérente de l'objet de la contestation.

\section{François : C'est pas du tout un nom bizarre ! C'est le nom d'un président américain récent, je te rappelle.}

(36) François a dit que c'était pas du tout un nom bizarre mais que c'était le nom d'un président américain récent et qu'il lui rappellait.

(37) François contredit que ce n'est pas du tout un nom bizarre. Il ajoute qu'il la rappelle que celui est un nom d'un président américain récent.

(38) François n'est pas d'accord et crie que «Bill» n'est pas du tout un nom bizarre. Il rappelle que c'est le nom d'un président américain récent.

(39) François s'est indigné et s'est crié que ce n'était pas du tout un nom bizarre et il a rappelé à Khoumba qu'au contraire, c'était le nom d'un président américain récent.

Il semble que l'intervention soit d'une facilité banale (le schéma «c'est»+ complément), toutefois la structure phrastique disloquée pose un problème important dans (36) ; dans (37), l'apprenant n'arrive pas à ajuster les pronoms, toutefois on peut le féliciter de sa tentative de résumer la valeur illocutoire de l'énonce à l'aide

\footnotetext{
${ }^{1}$ Pour dissiper la confusion entre l'énonciation principale et le métalangage, il serait peut-être judicieux d'ajouter des expansions aux SN : «le prénom de "Bill" » et «le mot "débile" »; on pourrait par ailleurs faire réfléchir l'apprenant sur l'anaphore de «François » dans la deuxième phrase ainsi que sur les moyens syntaxiques permettant d'assurer la cohérence de l'échange de façon explicite.
} 
du verbe « contredire » (même s'il n'en maîtrise pas la syntaxe). L'exemple (38) est le seul à être grammatical, mais le choix lexical du verbe «crier» est beaucoup moins réussi que celui de «s'indigner» dans (39) - verbe malheureusement coordonné à * «s'est crié », redondant.

XII. Khoumba : Pourquoi vous mettez pas « Aïssata » ou « Rachid » ou « Mohammed » ou ...

\section{Esméralda : Vous mettez tout le temps des noms de patos.}

(40) Khoumba a demandé pourquoi il mettait pas «Aïssata» ou « Rachid» ou «Mohammed » et Esméralda a ajouté qu'il mettait tout le temps des noms de patos.

(41) Khoumba a demandé pourquoi il mettait pas «Aïssata » ou « Rachid » ou «Mohammed ». Elle voulait continuer l'énumération mais Esmeralda lui a interrompu. Elle a dit que François mettait tout le temps des noms de patos.

(42) Khoumba a demandé donc pourquoi mettait-il pas «Aïssata» ou «Rachid» ou «Mohammed». Esméralda a dit qu'il mettait tout le temps des noms de patois [sic!].

(43) Khoumba semble changer de sujet en questionnant pourquoi le prof ne mettait pas un autre nom, comme «Aïssata» ou « Rachid» ou « Mohammed ». Esméralda est d'accord avec sa collegue en accusant le prof de mettre tout le temps des noms de patos.

(44) Khoumba ne s'est pas montrée convaincue et a demandé pourquoi François ne mettait pas p. ex. «Aïssata» ou «Rachid» ou «Mohammed». En ce moment, Esméralda a remarqué que François mettait tout le temps des noms de patos.

L'exemple (42) se distingue par rapport à (41) par la tentative de tenir compte des trois points («Elle voulait continuer l'énumération»). Dans (43), l'étudiant ressent un besoin de soigner la cohérence globale de la transposition de ce dialogue à caractère d'échange rapide, et propose un «donc» timide. Les derniers exemples sont beaucoup plus élaborés par rapport à l'original. Remarquons les reformulations comme «Khoumba semble changer de sujet», «Esméralda est d'accord avec sa collègue », « en accusant le prof », «Khoumba ne s'est pas montrée convaincue », « Esméralda a remarqué ».

XIII. François : C'est quoi des noms de ... ?

Esméralda : De babtous.

François : Babtous quoi?

Esméralda : C'est-à-dire de babtous, de Français, de «çaifran ».

(45) François a demandé ce que c'étaient ces noms et Esméralda a répondu que c'étaient des noms de babtous. François voulait savoir qui étaient Babtous et Esméralda a expliqué que de babtous, c'était de Français, des «çaifran ».

(46) François veut demander ce que c'est les noms de patos. Esméralda lui interrompt que ce sont des babtous. Francois demande ce que c'est. Esméralda explique que ce sont des babtous des Français, des « çaifran ». 
(47) François a demandé ce que c'était des noms de... Et Esméralda a terminé qu'il s'agit des babtous. François a encore une fois mal compris et a demandé quoi Baptous. Esméralda a dit que c'est-à-dire de baptous, de Français, des «çaifran ».

(48) François n'a pas compris ce que cela voulait dire « patos ». Esméralda a expliqué qu'il s'agissait de babtous, de Français, de «çaifran ».

L'auteur de (45) est très attaché à la structure phrastique de l'exemple de départ et la transpose à l'échelle $1: 1$ (notons quand même que l'étudiant a réussi à renoncer au « quoi » familier, contrairement à (10) ci-dessus), idem pour (46), sinon que le choix du verbe rend mal compte de la situation de communication observée : * «Esmeralda lui interrompt». Le (47) s'apparente au discours direct libre, et pèche de nouveau par son accumulation de structures syntaxiques parallèles. Le (48) finalement peut être jugé comme transposition synthétique et réussie de l'échange.

\section{François : Mais t'es pas Française toi, Esméralda ?}

Esméralda : Non, moi j'suis pas Française.

François : Ah bon ! J'étais pas au courant [ironique].

Esméralda : En fait, j'suis Française mais j'suis pas fière de l'être.

François : Moi, non plus je suis pas fier d'être Français.

(49) François lui a demandé si elle n'était pas Française et elle a repondu qu'elle n'était pas. François a constaté ironiquement qu'il n'était pas au courant. Esméralda a expliqué qu'elle était Française mais elle n'était pas fière de l'être. François était de même avis (...).

(50) (...) Esméralda a dit qu'en fait, elle était Française mais elle n'était pas fière de l'être. François était d'accord avec elle et que lui aussi, il n'était pas fier d'en être.

(51) François a dit qu'elle n'est pas Française et Esméralda était d'accord. (...).

(52) (...) Esméralda admet qu'elle est Française mais qu'elle n'est pas fière de l'être. François admet qu'il n'est pas fier d'être Français non plus.

(53) (...) Esméralda a précisé qu'elle est Française mais elle n'est pas fière d'être Française. François a dit qu'il n'est plus fier de l'être.

(54) François s'assurait que Esmeralda n'a été pas Française. Elle est d'accord avec lui. François ironise qu'il n'était pas au courant. Esmeralda ajoute qu'elle en fait est Française mais elle n'est pas fière de l'être. Tous rigolent.

(55) (...) Esméralda éclairci qu'elle est Française mais elle n'en est pas fière.

(56) François lui a pourtant demandé, toujours étonné, si elle n'était pas Française. Esméralda a répondu par la négative. François a répondu ironiquement qu'il n'était pas au courant. Esméralda a réagi en disant qu'en fait elle était Française mais elle n'était pas fière de l'être. François a dit que lui aussi, il n'était pas fier d'être Français non plus.

La didascalie introduisant l'adjectif « ironique » est d'une grande utilité pour reformuler l'intervention de François $(49,54,56)$. En revanche, l'expression « être de même avis » dans (49) est ambiguë puisqu'on n'est pas sûr de la raison du consensus : ne pas être fier ou être Français ? (observons entre parenthèses un essai de 
désambiguïsation maladroite dans (50)). Dans (51), l'étudiant se méprend sur l'intention de François («François a dit qu'elle n'est pas Française ») puisque dans l'interaction au discours direct, celui-ci ne constate pas, il pose la question ( «Mais t'es pas Française toi, Esméralda ? ») et s'étonne. L'exemple (52) est réussi dans la reformulation de l'intervention d'Esméralda par la structure lexico-grammaticale «admettre que..., mais...»; toutefois la charpente de la phrase défaille après «mais» (un nouveau verbe, par exemple «objecter», y ferait défaut). Le deuxième « admettre » (ayant « François » pour sujet) perd son impact illocutoire du fait de la répétition. Dans (53), par manque de compétence grammaticale ou par inadvertance, l'étudiant aboutit à un contresens («ne plus être fier» vs «ne pas être fier non plus»). Dans (54), l'intention pragmatique est viciée par l'imparfait («s'assurait», impliquant l'aspect itératif, absent de l'énoncé de François). Il est toutefois à remarquer que l'étudiant fait partie des rares qui tiennent compte du contexte en résumant aussi le non-verbal («Tous rigolent»). Dans (55), l'étudiant prend le risque d'utiliser un verbe plus rare, dont le choix serait à discuter. Finalement, l'exemple (56) se distingue par une plus grande implication de ce qu'on classe dans les barèmes de correction dans le critère « reformulation personnelle », visible dans les constructions phrastiques plus élaborées (propositions participiales, gérondif), l'ajout de connecteurs logiques («pourtant»), les verbes performatifs («a réagi»), mélangés toutefois avec des phrases très simples du point de vue syntaxique («François a dit que... ») et des répétitions des mêmes lexèmes («a dit », « en disant »; «a répondu » en tête de deux phrases consécutives).

XV. Khoumba : Mais pourquoi vous mettez toujours des noms comme ça alors?

François : Mais enfin Khoumba, tu comprends bien que si je choisis à chaque fois des prénoms en fonction des origines diverses qu'il y a dans cette classe, j'vais pas m'en sortir.

Esméralda et Khoumba (en même temps) : Ben, changez un peu !

(57) (...) François a répondu que Khoumba comprenait bien que s'il choisissait à chaque fois des prénoms en fonction des origines diverses qu'il y avait dans cette classe, il allait pas s'en sortir.

(58) Esméralda et Khoumba ont dit de changer un peu.

(59) (...) Esméralda et Khoumba ont dit en même temps que ben, changiez un peu.

(60) (...) Esméralda et Khoumba lui ont conseillé de changer un peu.

Les transpositions ci-dessus sont souvent effectuées de façon mécanique, en respectant les règles grammaticales (57-58). L'exemple (59) est flagrant de ce point de vue car il révèle que l'étudiant n'a pas assimilé la compétence pragmatique qui devrait lui permettre de ranger «ben» dans la classe des phatèmes, éléments inappropriés dans la narration. L'exemple (60) a le mérite d'aller droit au but, en reformulant habilement l'intention des deux collégiennes à l'aide du verbe « conseiller» . 
XVI. François : Alors, qu'est-ce que tu me proposes ? [à Khoumba]

Khoumba : Aïssata ! Euh ... Fatou, non ... euh ...

Esméralda et Khoumba (ensemble) : Aïssata !

(61) François a demandé quoi Khoumba lui proposait. Il a repondu que « Aïssata », puis « Fatou » et à la fin Esméralda et Khoumba ont dit ensemble « Aïssata ».

(62) (...) Khoumba a répondu qu'Aïssata ou Fatou et les deux filles ensemble ont crié qu'Aïssata.

(63) (...) Khoumba crie que Aïssata mais elle hésite, elle ajoute le nom Fatou aussi. En même temps, Esméralda et Khoumba exclament qu'elles veulent Aïssata.

(64) (...) Khoumba a crié Aïssata. Elle a aussi proposé Fatou mais elle n'était pas vraiment décidée. Esméralda et Khoumba ont dit ensemble Aïssata.

(65) (...) Khoumba crie que Aïssata, puis elle hésite et dit «Fatou ». Enfin Khoumba et Esméralda se décident et crient ensemble que Aïssata.

Dans la plupart de ces exemples, les étudiants s'embrouillent en essayant d'être exhaustifs et de rendre compte des hésitations des collégiennes (ex. 61). En plus, la rapidité de l'échange, ressentie même à partir de la transcription, n'est pas aisée à transposer dans une narration. Il est difficile de décider laquelle des réponses grammaticales de cette série est la plus acceptable : (62), (63), (64) ou (65) ? - et ce, notamment parce que la cohésion est assurée surtout par la coordination à l'aide de « et » et « mais » (la plus soignée peut-être dans 139, cf. aussi le choix des performatifs « hésiter », « se décider »).

Pour terminer, force est de constater que, afin d'obtenir des données plus fiables, nous aurions dû comparer les résultats de cette transposition effectuée en classe avec un devoir réalisé à la maison, étant donné que parfois, nous avons remarqué que l'étudiant cherchait à produire une réponse élaborée, mais y faisait apparaître des solutions de facilité - un symptôme révélateur, peut-être, du manque de temps qui serait à l'origine de la faible qualité de certaines productions. Quoi qu'il en soit, en regard des attentes claires des concepteurs d'exercices de transposition du discours direct en discours indirect, il transparait déjà que dans le cas des textes authentiques, la méthodologie d'apprentissage de cette question grammaticale (signalée au début du présent article) nécessite d'être enrichie par l'inclusion des éléments de compétence pragmatique afin d'obtenir non seulement des transformations syntaxiquement acceptables, mais aussi, ayant du sens. En témoignent certains problèmes relevés concernant: la distinction des plans énonciatifs (exemple I ci-dessus), la cohésion (I), la prise en compte de la valeur illocutoire des énoncés, en relation avec la ponctuation (dans chaque exemple, mais notamment dans II, III, VI, XII, $\mathrm{XVI})$ et le choix des verbes introducteurs compte tenu des didascalies/du contexte (VIII, IX), la gestion du passage de l'oral à l'écrit, y compris les phatèmes (II, XV), et du registre familier au registre standard (V, VI), la syntaxe de l'oral, surtout en ce qui concerne les structures disloquées (III, IV, VIII, XI), la progression thématique (VII), les anaphores et la création de chaînes de référence (IX), la gestion des temps 
verbaux (IX), l'insertion des éléments interprétatifs, notamment des performatifs provenant de l'énonciateur (X), l'acceptabilité des structures, impliquant par exemple le fait d'éviter l'accumulation de propositions simples et de favoriser les constructions synthétiques contenant des gérondifs ou des propositions participiales (XIII, XIV), une désambiguïsation du sens (XIV), la prise en charge du non-verbal (XIV), les connecteurs logiques et d'autres procédés de cohésion (XIV, XVI).

\section{TRANSFORMATION DU DIALOGUE EN RÉCIT}

Le corpus se compose de 17 copies, dont 12 de IIIL et 5 de IM. Nous l'analyserons à travers certaines caractéristiques du texte narratif en nous référant à la typologie de J.-M. Adam (1997), sans prendre en compte toute la complexité du récit dans la perspective didactique d'une tâche de production en corrélation avec les étapes de développement de la compétence de communication, puisque, comme l'argumente U. Paprocka-Piotrowska (2013, p. 291), « il semble que dans le cas d'un apprenant adulte, les connaissances linguistiques générales sur l'organisation des langues et la communication ainsi que les connaissances générales sur le mode peuvent servir de pivot solide pour la construction d'un récit en L2 ».

\subsection{QUESTION DE POINT DE VUE ET DE MISE EN SCÈNE}

(66) Un jour, pendant le cours de français, j'étais témoin d'une discussion très intéressante.

(67) Un jour, dans un lycée multiethnique en banlieu de Paris, le professeur du français discutait avec ses élèves.

(68) Les cours ont commencé. Le professeur du français écrit une phrase sur le tableau, avec un mot « succulent ».

Les phrases citées ci-dessus activent chacune un invariant textuel : journal intime/anecdote personnelle dans (66) à travers le «je » de l'énonciateur ; récit stéréotypé avec la formule «un jour» dans (67), au contraire de (68) qui introduit l'histoire in medias res: "Les cours ont commencé ». La diversité de ces incipit prouve que la médiation d'un dialogue n'est pas un procédé anodin et impose dès le début le choix d'une option narrative.

\subsection{REFORMULATION DE L'ENJEU DE L'INTERACTION}

(69) François, notre prof, a essayé de nous expliquer un mot « succulent».

(70) L'enseignant François veut faire une phrase avec «succulent » et il écrit la phrase «Bill déguste un succulent cheeseburger ». Un des élèves pense que la phrase avec ce mot n'est pas bonne. L'élève qui s'appelle Cherif parle avec François. 
(71) Tout de suite, cette phrase a engendré des controverses.

(72) La querelle s'est commencé quand François a écrit sur le tableau la phrase expliquant la signification d'un mot «succulent». Il a utilisé les mots américains : « Bill » et « un cheeseburger». Les élèves ont commencé de lui critiquer.

(73) Aujourd'hui François, comme toujours, dirige les classes, il écrit au tableau la phrase « Bill déguste $(. .)$.$» .$

(74) Pendant l'un de ses courses, il voulait expliquer aux ses élèves qu'est-ce que ça veut dire «succulent» en se servant d'un exemple d'usage ce mot dans la phrase «Bill déguste $(. .)$.$» .$

(75) Ce phrase cause plusieurs problèmes. Premièrement Cherif qui est l'un des élèves, ne se plaisait pas cette exemple (...). Le deuxième problème de ce phrase était un prenom qui a été utilisé par François.

(76) Un jour, pendant le cours de français, François, le professeur, écrivit une phrase au tableau: "Bill déguste un succulent cheeseburger». La phrase avait pour but d'explique le mot «succulent». Malheureusement la référence aux USA n'a pas plût aux élèves de la classe, ils commencèrent à critiquer les mots qui se trouvaient dans la phrase

(77) Le professeur du français écrit une phrase sur le tableau, avec un mot « succulent». Cela n'a pas provoqué l'enthousiasme de la classe. En effet, les élèves se sont mis à discuter et à se disputer avec le prof.

(78) François a écrit une phrase au tableau «Bill déguste (...) ». Puisque les élèves ne sont pas sages, ils font des remarques hors sujet.

Dans les exemples ci-dessus, nous pouvons aussi observer plusieurs procédés d'ouverture d'un texte narratif (cf. par ex. 73 et $69^{2}$ ). Nous les avons sélectionnés dans la diversité des reformulations du conflit entre le professeur et la classe. En comparant (69) et (74) aux exemples (1-2) de la section antérieure, force est de constater que la valeur illocutoire est mieux saisie dans les deux premiers à travers les reformulations : «essayer d'expliquer », «vouloir expliquer » ${ }^{3}$. Dans (72) apparaît l'idée de «querelle » (à défaut d'un meilleur mot sans doute), mais aussi un complément de sens sous forme de l'hyperonyme «les mots américains », rendant visible le processus de conceptualisation de la situation présentée dans le film par l'étudiant. Le mot «critiquer», dans la suite, peut être discuté comme choix lexical, il est toutefois intéressant du fait qu'il annonce la suite de l'interaction. Le problème lexical est mieux résolu dans la phrase (77) où «les élèves » se mettent à « discuter » («se disputer avec le prof », qui suit, est déjà moins intéressant du fait des statuts inégaux des participants de la «dispute », même si la situation verbale s'en rapproche effectivement). Remarquons aussi dans le même exemple la belle litote

\footnotetext{
${ }^{2}$ Qui pose d'ailleurs «notre prof » et « François » comme deux formes nominales d'adresse sur le pied d'égalité - contrairement à l'usage pragmatique puisqu'un professeur en délocution devrait être repris par la forme nominale «Monsieur»+ patronyme.

${ }^{3}$ Seul l'exemple (70) semble être très proche des réponses classées dans la section 3 du présent article.
} 
« cela n'a pas provoqué l'enthousiasme de la classe », ainsi qu'une autre formulation soignée de l'apprenant dans (71): «cette phrase a engendré des controverses ». Dans (75), nous avons affaire au procédé de classement des problèmes décrits en deux types, balisés dans le texte par l'adverbe «premièrement» et le $\mathrm{SN}$ «le deuxième problème ». L'exemple (76) contient le modalisateur «malheureusement », signe de subjectivité de l'auteur, et aussi d'autres reformulations généralisantes très explicites en ce qui concerne le conflit: "la référence aux USA », «ils commencèrent à critiquer les mots ${ }^{4}$. Finalement, l'exemple (78) semble conceptualiser la situation du point de vue non d'un observateur externe, mais du professeur lui-même, à travers le choix lexical de l'adjectif « sages ».

\subsection{PÉRIPÉTIES ET FIN}

Cette partie rend à notre avis le mieux compte de la différence entre les productions correspondant aux deux consignes.

(79) Pour nous faciliter la compréhension de cette notion, il nous a donné un exemple d'utilisation de ce mot en une phrase : «Bill déguste un succulent cheeseburger ». Et voilà. Cela, c'était le début d'une vive échange de pensées. Immédiatement, Cherif a protesté (...). François a expliqué (...). Cette fois-ci, c'était Angélica qui a réagi. (...) François nous l'a expliqué. Mais, ça n'était pas la fin. Khoumba a demandé (...). La discussion a éclaté encore une fois.

(80) Selon Esméralda et Khoumba il devrait changer le nom de garçon qui « déguste un succulent cheeseburger ». Les filles proposent le nom « Aïssata ».

(81) La discussion a été finie par la proposition des filles de remplacer le prénom par « Aïssata».

(82) Mais finallement le prof a demandé l'opinion de ses élèves et ils lui ont donné quelques propositions.

(83) Finalement François permet les élèves choisi le nom.

(84) Esméralda et Khoumba ont lui suggéré un changement.

(85) Finalement, il décida de changer le prénom en Aïssata.

(86) Les étudiants ont préféré que le prof emploie des noms propres à des cultures africaines.

(87) Après l'un des élèves demande pourquoi il utilise les noms, selon lui, bizarres comme « Bill » et pas «Aïssata » ou « Rachid».

(88) François finalement est d'accord et attend des propositions.

L'exemple (79) se caractérise par sa charpente narrative élaborée. L'étudiant introduit des connecteurs et résume les performances verbales de certains locuteurs («Cherif a protesté », «Angélica a réagi», «la discussion a éclaté encore une fois »).

\footnotetext{
${ }^{4} \mathrm{Cf}$. l'exemple (72) où les élèves ont commencé à $*_{«}$ lui critiquer ».
} 
La dernière partie du texte est aussi présentée de façon beaucoup plus concise, cf. les exemples (80-86). L'exemple (87) contient une manipulation intéressante, en abandonnant l'identité des élèves en faveur de la mise en relief de l'enjeu du conflit. L'exemple (88) valorise l'accord du professeur, en reléguant les propositions de prénoms au deuxième plan, ce qui est aussi digne d'attention comme processus cognitif révélateur de la réorganisation personnelle des données par l'auteur de la médiation.

\subsection{ESSAI D'INFÉRER UN SENS À L'INTERACTION TRANSFORMÉE}

(89) Il s'est avéré que les élèves ne se sentaient pas Français parce qu'ils étaient originaires d'ailleurs p. ex. de l'Afrique. Donc nous avons passé au sujet de la conscience ethnique. Incroyable ! Et tout cela faute à un cheeseburger... On peut constater que la vie d'un professeur n'est pas facile, mais elle est sans doute intéressante.

(90) Après le cours François était un peu désespéré.

Il est intéressant d'observer que certains étudiants ressentent un besoin d'interpréter le sens de toute la séquence interactive en ajoutant dans leur texte une morale, comme dans (89), ou un épilogue, comme dans (90).

\section{5. ÉVALUATION FINALE DE LA TRANSFORMATION DU DIALOGUE EN RÉCIT}

Du point de vue de la mise en page, le texte produit en réaction à cette consigne se trouve à l'opposé exact du texte transformé en discours indirect qui était morcelé à l'extrême. La plupart des narrations ne contiennent aucune subdivision en paragraphes, sauf quelques exceptions qui divisent le texte en trois parties (ce qui est peutêtre le résultat d'un conditionnement didactique : un texte doit se composer « obligatoirement » d'une introduction, d'un développement et d'une conclusion). Seule une copie de la deuxième série aurait être pu insérée parmi les copies de la première ; la plupart présentent un choix de reformulations personnelles éloignées du texte de base et rendent compte de la dynamique pragmatique de l'interaction présentée.

Les exemples suivants témoignent d'une compétence discursive des locuteurs correspondant bien au niveau de compétence du programme. Certes, cette compétence discursive est tributaire de la compétence lexicale (plus avancée dans 91 que dans 92, cf. aussi 93 et 94 - en dépit des formes verbales erronées), syntaxique (cf. 95) ou socioculturelle (connaissance du registre, cf. 96), mais, élicitée par la consigne, elle donne en résultat un texte plus acceptable que dans le cas d'une activité à objectif opérationnel purement grammatical : 
(91) Puis le professeur a utilisé l'expression idiomatique qui était incompréhensible (...).

(92) Ensuite, une élève qui s'appelle Khoumba demande pourquoi l'enseignant a utilisé le prénom « Bill », parce qu'il est bizarre. Pour François ce prénom est normal.

(93) Un problème était solutioné mais un autre se produit.

(94) À ce moment-là, Esméralda a joigné à la discussion.

(95) François, qui avait ce type de problème assez souvent, explique que la remarque de Cherif est très bonne, et que ça peut, les élèves, mettre la puce à l'oreille. Les étudiants ne comprenaient pas cette expression. François les a expliqué alors que « mettre la puce à l'oreille » signifie « donner un indice ». Puis il a expliqué comment Cherif lui a aidé a montrer voir ce que signifie « succulent ».

(96) Cherif a dit qu'il s'en fichait, mais en tout cas un cheeseburger, c'était pas bon.

La contrepartie d'une plus grande liberté dans le traitement du texte est la production de contresens, comme dans les exemples qui suivent :

(97) Sa copine, Esméralda dit qu'il enumere seulement les noms des immigrés d'Algérie française.

(98) L'enseignant explique qu'il utilise des prénoms en fonction des origines diverses ${ }^{5}$.

(99) L'un des élèves, Cherif, dit que le mot anglais « cheeseburger » n'est pas bon et il demande pourquoi le professeur utilise ce mot.

(100) Khoumba répond « Aïssata », mais elle change d'avis en disant le baragouin (...) .

Ces contresens semblent les seuls éléments de réponse susceptibles d'être réellement pénalisés lors d'une évaluation formative.

\section{CONCLUSION}

La présente étude a laissé entrevoir deux facettes de l'activité de médiation intralinguistique : elle peut avoir pour objectif opérationnel la pratique des éléments de la langue (notre première consigne) ou la formation de la compétence discursive en communication authentique (notre deuxième consigne). Il est évidemment abusif de constater qu'a priori, l'une est inutile et l'autre louable: les défaillances de la seconde série de copies montrent avec force à quel point la qualité des productions examinées sous l'angle de la compétence discursive dépend des compétences langagières de l'apprenant ${ }^{6}$. Le problème que nous voudrions en revanche mettre en relief est le manque de passage didactisé de l'une vers l'autre, tout en observant que rapporter les paroles est un besoin naturel des apprenants à partir du niveau $\mathrm{A}$.

\footnotetext{
${ }^{5}$ Dans l'interaction, il objecte qu'il ne saurait le faire.

${ }^{6}$ Et pas seulement. Comme le constate Zając (2004, p. 102), «il s'ensuit que l'apprenant développe sa compétence discursive parallèlement avec d'autres compétences, elle "grandit" en quelque sorte au fur et à mesure que grandissent ses capacités strictement langagières ou sociolinguistiques ainsi que ses différents savoirs (savoir du monde, savoir-être, savoir-faire, savoir-apprendre) ».
} 
Ils entreprennent régulièrement l'effort de raconter un dialogue (ne serait-ce que celui qui sert de support au cours) en s'engageant dans des structures du type «il a dit que... » et en déroutant le professeur qui se sent obligé de corriger toutes les fautes dues au savoir grammatical déficient du débutant. Cependant, une fois la technique enseignée, le programme n'y revient que rarement, et dans la plupart des cas, seulement à partir de dialogues écrits en langue standard, bien structurés, avec de grandes parties de monologues, dont les apprenants font des comptes rendus ou des résumés. Par conséquent, en classe de FLE, le discours indirect, réduit à un exercice technique, est extrêmement éloigné de sa pratique sociale omniprésente, ce qui résulte probablement de sa subordination à des finalités qui relèvent de l'évaluation (il est plus aisé de donner des points pour des transformations syntaxiques isolées que pour un texte produit à partir d'un autre texte).

Les questions qui se posent à la suite de cette étude, qui - espérons-le, a prouvé le besoin de la dominante pragmatique dans l'enseignement de la transformation des dialogues en textes narratifs - sont donc les suivantes :

- Quels doivent être les objectifs de l'enseignement du discours indirect par rapport aux enjeux de la démarche actionnelle?

- Quels textes de base choisir? Quelle place réserver au discours oral par rapport à l'écrit (nettement privilégié) ? Dans quel registre ? Comment didactiser le passage d'un registre à l'autre?

- Comment élaborer les critères d'évaluation d'une activité de médiation entre le dialogue et le récit ? Comment équilibrer la place de la grammaticalité et de la reformulation compte tenu des actes de parole et des valeurs illocutoires et perlocutoires des énoncés ?

- Comment travailler l'arrimage référentiel (et notamment les anaphores), événementiel (les connecteurs logiques), énonciatif (les enjeux de l'énonciation de discours et de l'énonciation historique) et informatif (la saillance des informations nouvelles, cf. Gagnan, 2015) des informations lors de la construction d'un texte construit au discours indirect?

- Comment mettre en valeur l'aspect formatif de la transposition d'un dialogue en un autre type de texte (partant du principe que la grammaticalité ne peut pas être le seul critère d'évaluation et que les contresens sont les seuls éléments méritant d'être pénalisés)? 


\section{BIBLIOGRAPHIE}

Adam, J.-M. (1997, nouv. éd. 2008 et 2011). Les textes : types et prototypes. Paris : Armand Colin.

Bégaudeau, F., Cantet, L., Campillo, R. (2008). Le scénario du film «Entre les murs ». Paris : Gallimard.

Biardzka, E. (2009). Les échos du Monde. Pratiques du discours rapporté dans un journal de la presse écrite. Wrocław : Wydawnictwo Uniwersytetu Wrocławskiego.

Cadre commun européen de référence pour les langues : apprendre, enseigner, évaluer. (2001). Strasbourg : Didier.

Callet, S. (2012). Les discours direct et indirect. Louvain : De Boeck/Duculot.

Gagnon, O. (2015). Travailler la cohérence du texte. In F. Boch, C. Frier (réd.), Écrire dans l'enseignement supérieur. Des apports de la recherche aux outils pédagogiques (pp. 111-149). Grenoble : ELLUG.

Mennesson, A., Entre les murs de Laurent Cantet. URL : http://docplayer.fr/16094991-Entre-les-mursde-laurent-cantet-dossier-realise-par-alice-mennesson.html

Paprocka-Piotrowska, U. (2013). Construction du récit dans la communication en langue étrangère. Lublin : Werset.

Traverso, V. (1996). La conversation familière. Lyon : Presses Universitaires de Lyon.

Zając, J. (2004). Du communicatif au discursif. Warszawa : Instytut Romanistyki Uniwersytetu Warszawskiego. 